\title{
The Effect of Bcr-Abl Protein Tyrosine Kinase on Maturation and Proliferation of Primitive Haematopoietic Cells
}

\author{
Anne-Marie Buckle, ${ }^{1}$ Rachel Mottram, ${ }^{1}$ Andrew Pierce, ${ }^{1}$ Guy S. Lucas, ${ }^{2}$ \\ Nigel Russell, ${ }^{3}$ Jaleel A. Miyan, $^{1}$ Anthony D. Whetton ${ }^{1}$ \\ ${ }^{1}$ Leukaemia Research Fund, Department of Biomolecular Sciences, University of \\ Manchester Institute of Science and Technology, Manchester, U.K. \\ ${ }^{2}$ Department of Clinical Haematology, Manchester Royal Infirmary, \\ Manchester, U.K. \\ ${ }^{3}$ Dept of Haematology, Nottingham City Hospital, Nottingham, U.K. \\ Accepted June 30, 2000.
}

\begin{abstract}
Background: Chronic Myeloid Leukaemia (CML) is characterised by the chromosomal translocation resulting in expression of the $\mathrm{Bcr}-\mathrm{Abl}$ protein tyrosine kinase (PTK) in early stem cells and their progeny. However the precise nature of Bcr-Abl effects in primitive CML stem cells remains a matter of active debate.

Materials and Methods: Extremely primitive BcrAbl fusion positive cells were purified from patients with CML using multiparameter flow cytometric analysis of CD34, Thy, and lineage marker (Lin) expression, plus rhodamine-123 (Rh-123) brightness. Progenitor cells of increasing maturity were examined for cycling status by flow cytometry and their proliferative status directly correlated with cell phenotype. The activation status of a key transcription factor, signal transducers and activators of transcription (STAT-5), was also analyzed by immunocytochemistry. Results: The most primitive stem cells currently defined (CD34+Lin-Thy + Rh-123lo) were present as a lower proportion of the stem cell compartment
\end{abstract}

(CD34+Lin-) of CML patients at presentation than of normal individuals $(2.3 \% \pm 0.4$ compared with $5.1 \% \pm 0.6$ respectively). Conversely there was a significantly higher proportion of the more mature cells (CD34+Lin-Thy-Rh-123 hi) in CML patients than in normal individuals $(79.3 \pm 1.8$ compared with $70.9 \pm 3.3)$. No primitive subpopulation of CML CD34+Lin- cells was cycling to a significantly greater degree than cells from normal donors, in fact, late progenitor cells (CD34+Lin + ) were cycling significantly less in CML samples than normal samples. STAT5, however, was observed to be activated in CML cells.

Conclusions: We conclude that no subpopulation of CML stem cells displays significantly increased cell cycling. Thus, increased cycling cannot be a direct consequence of Bcr-Abl PTK acquisition in highly enriched stem cells from patients with CML. In vivo CML need not be considered a disease of unbridled stem cell proliferation, but a subtle defect in the balance between self renewal and maturation.

\section{Introduction}

Chronic Myeloid Leukaemia (CML) is a haemopoietic stem cell disease characterized by a specific chromosomal translocation that leads to the production of the chimeric protein, $\mathrm{Bcr}-\mathrm{Abl}(1,2)$. $\mathrm{Bcr}-\mathrm{Abl}$ is a promiscuous protein

Address correspondence and reprint requests to: A-M Buckle. Leukaemia Research Fund, Department of Biomolecular Sciences, UMIST, Manchester, U.K. Phone: 0161-200-4214; Fax: 0161-236-0409. a.buckle@umist.ac.uk tyrosine kinase (PTK) that causes a leukaemialike disease in mouse models (3-8). Bcr-Abl PTK has been studied in a variety of different cell systems, including fibroblasts, interleukin3 (IL-3) dependent haemopoietic cell lines that are differentiation-blocked, and also in haemopoietic cell lines that are able to differentiate (9-19). Within both fibroblasts and IL-3dependent, differentiation-blocked cell lines, Bcr-Abl can stimulate autonomous proliferation in that the cells do not require serum factors or 
cytokines, respectively, in order to proliferate. However, others $(14,20)$ have demonstrated that the initial effects of Bcr-Abl PTK are not to induce cytokine-independent survival and proliferation. Most recently, it has been shown that purified populations of primitive cells derived from CML patients can proliferate in the absence of added growth factor by virtue of their autocrine production of IL-3 and granulocyte-colony stimulating factor (G-CSF) (21). These studies were performed either on cell lines or on cultured cells from CML patients; whereas, the precise nature of the Bcr-Abl effect on stem cell proliferation in vivo has not been investigated due to difficulties with performing multiparametric analyses to define the stem cell compartment and simultaneously analyze the cell cycle status.

The observation that the Philadelphia $(\mathrm{Ph})$ chromosome can be found in many different haematopoietic lineages has been held to infer that the initial Bcr-Abl translocation event occurs in primitive multipotent stem cells and acquisition of Bcr-Abl has been demonstrated in all populations of stem cells investigated so far, including those with the most primitive phenotype $(22,23)$. As fluorescence in situ hybridization (FISH) technology has become more sophisticated and accurate, almost all CML progenitors have been found to be Bcr-Abl fusion-positive at presentation $(24,25)$. However, although it is clear that Bcr-Abl acquisition by stem cells is an essential transforming event implicated in the pathogenesis of CML, the mechanism underlying transformation as a consequence of elevated kinase activity has yet to be clearly defined. Since the Bcr-Abl translocation is known to occur in the stem cell compartment of CML patients, it is the expression of Bcr-Abl in the context of this specific cell type that will provide biologically relevant information on the mechanism underlying the leukaemogenic effects of Bcr-Abl in CML and the development of novel treatment strategies. In the present study, we assessed the maturation and cycling status of Bcr-Abl PTK positive cells in primary, uncultured stem cell populations isolated directly from patients with CML.

\section{Materials and Methods}

\section{Primary Cells}

Samples of peripheral blood from chronic phase Chronic Myeloid Leukemia (CML) patients were collected at presentation, before treatment. All samples used in the study were shown to be Philadelphia positive by standard cytogenetics, and patients with additional chromosome abnormalities were excluded from the study. Mononuclear cells (MNC) were prepared by centrifugation over Ficoll Hypaque (Pharmacia Biotech, Herts, UK), and in some cases CD34+ cells were selected using the MiniMacs system (Miltenyi Biotech Ltd., Bisley, UK). Cells were then stained with CD34 allophycocyanin (APC), fluorescein isothiocyanate (FITC) conjugated lineage markers (CD2, CD14, CD 15, CD 19,) and Thy-1 phycoerythrin (PE) (all from Becton Dickinson, Oxford U.K.). Negative controls using isotype-matched antibodies were routinely performed and dead cells were excluded by propidium iodide staining. Stem cells were isolated by multiparameter sorting on a FACSVantage (Becton Dickinson, Oxford, U.K.), with purities of $>90 \%$ (Fig. 1).

\section{FISH Analysis}

Slides were prepared either by sorting cells directly onto microscope slides using the FACSVantage CloneCyt software (Becton Dickinson, Oxford, U.K.), or by preparation of cytospins from bulk-sorted cells. Slides were air-dried overnight and stored at $-80^{\circ} \mathrm{C}$ until use, then fixed in 3:1 methanol:acetic acid at room temperature for $30 \mathrm{~min}$, before dehydration in alcohol. Slides were digested using $10 \mu \mathrm{g} / \mathrm{ml}$ proteinase $\mathrm{K}$, dehydrated in alcohol, then soaked in $2 \mathrm{X}$ standard saline citrate (SSC) pH 7.0/0.5\% NP40 for $2 \mathrm{hr}$ at $37^{\circ} \mathrm{C}$. Cells were denatured in formamide for $5 \mathrm{~min}$ at $73^{\circ} \mathrm{C}$, then dehydrated in alcohol. The BCR/ABLl D-FISH ${ }^{\mathrm{TM}}$ translocation DNA probe (Oncor Inc., Middlesex, UK) was denatured at $72^{\circ} \mathrm{C}$ for $5 \mathrm{~min}$ before adding to slides that were then hybridized overnight at $37^{\circ} \mathrm{C}$. After washing slides in $0.5 \mathrm{X} \mathrm{SSC}$ for $5 \mathrm{~min}$ at $70^{\circ} \mathrm{C}$, slides were mounted in Vectorshield (Vector Laboratories, Peterborough, UK) and analyzed on a Leica fluorescence microscope. Using this probe, normal cells displayed a pattern of two red signals and two green signals. Abnormal cells displayed a pattern of one red signal, one green signal, and two fusion signals, or two red signals, two green signals, and one fusion signal. Nuclei displaying any other signal patterns were not scored.

\section{Cell Cycle and Rh-123 Analysis}

Sorted normal bone marrow (NBM) and CML cells were incubated with Rhodamine-123 (Rh-123) at $0.1 \mu \mathrm{g} / \mathrm{ml}$ for $15 \mathrm{~min}$ at $37^{\circ} \mathrm{C}$, then washed and incubated in Hoechst 33342 (Mol- 

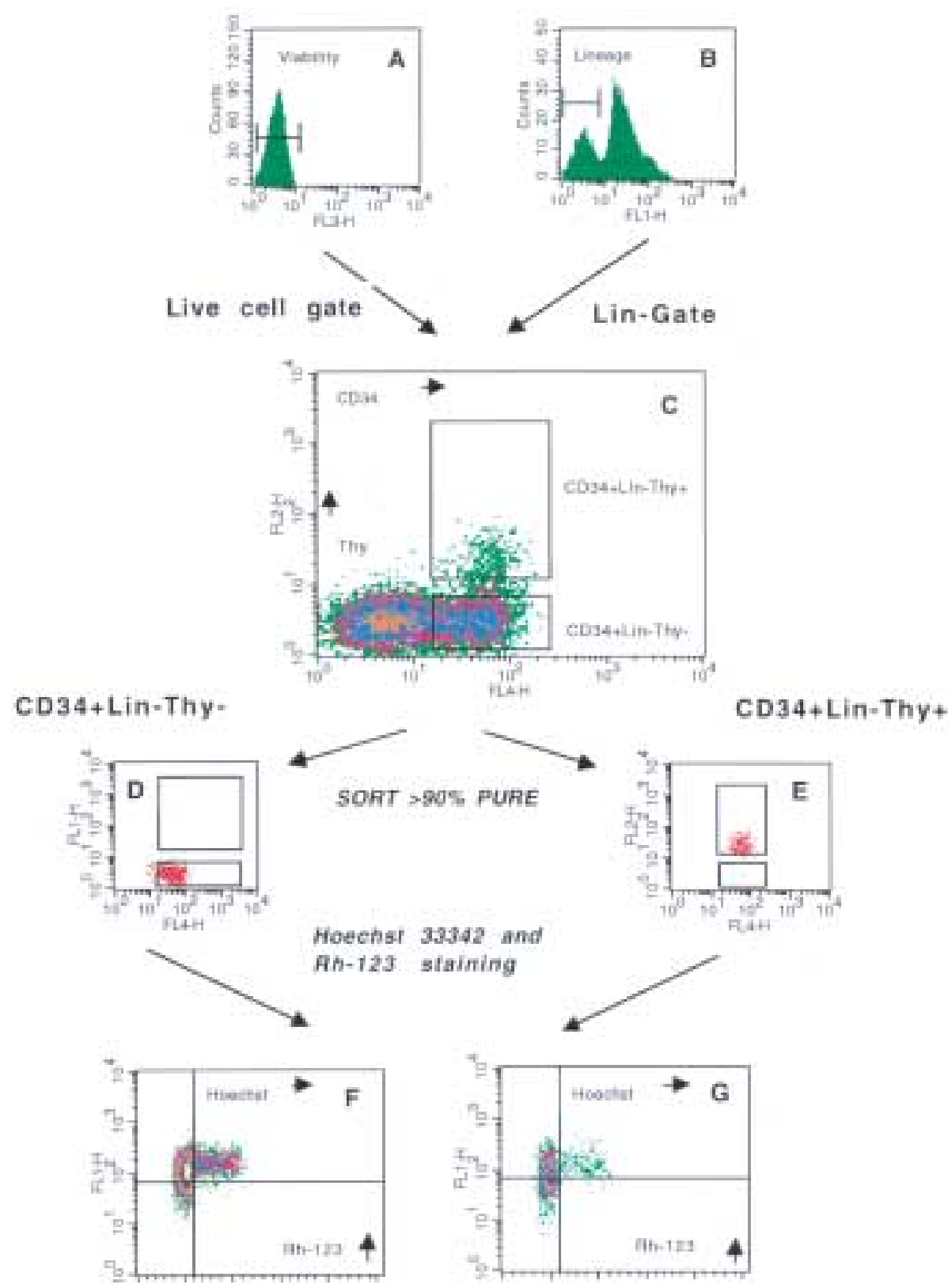

Fig. 1. Strategy for flow cytometric analysis of normal bone marrow (NBM) and chronic myeloid leukaemia (CML) samples. Mononuclear cells were stained with CD34+, lineage marker, and Thy antibodies as described in "Materials and Methods." Gates were set to sort live (propidium iodide excluding), lineage-negative cells as shown (A and B). Further gating, as shown in (C) was applied to define the CD34+Lin-Thy+ and CD34+Lin-Thypopulations revealed by staining with CD34 allophycocyanin (APC) and Thy phycoerythrin (PE). Using this four-parameter approach, CD34+Lin-Thy-

ecular Probes, Leiden, Holland) (10 $\mu \mathrm{M})$ for 45 min at $37^{\circ} \mathrm{C}$. Cell cycle status and Rh-123 retention were evaluated on the FACSVantage flow cytometer using ultraviolet (UV) and 488
(D) and CD34+Lin-Thy + (E) populations were sorted to $>90 \%$ purity. The sorted cells were then stained with rhodamine-123 (Rh-123) and Hoechst 33342 to simultaneously analyze the cell cycle and Rh-123 retention profiles of the sorted populations (F) and (G). The quadrant marker separates high levels of rhodamine-123 (Rh-123hi) from low levels of rhodamine-123 (Rh-123lo) cells and cycling cells from non cycling cells. A sample of normal bone marrow is depicted throughout this figure; cells from CML patients were processed using an identical strategy.

excitation, respectively. Fluorescence-activated cell sorting (FACS) data was analyzed using CellQuest software (Beckton Dickinson, Oxford, UK) (Fig. 1). 


\section{STAT 5 Translocation}

Slides of CD34+ cells were prepared by sorting 3000-5000 cells directly onto microscope slides using the FACSVantage CloneCyt software. Cytospins were prepared of mononuclear cells. Slides were air-dried, fixed in methanol $\left(10 \mathrm{~min}\right.$ prechilled to $\left.-20^{\circ} \mathrm{C}\right)$, then allowed to dry at room temperature. Slides were rehydrated in phosphate-buffered saline/ bovine serum albumin (PBS/BSA; $1 \%$ )/Tween $20(0.05 \%)$, stained with a mouse monoclonal antibody to (STAT-5; Santa Cruz, Santa Cruz, CA) for $40 \mathrm{~min}$ at room temperature, washed, then incubated with FITC anti-mouse (Santa Cruz) for a further $40 \mathrm{~min}$. Nuclei were visualized using propidium iodide staining. Isotype controls for STAT-5 were routinely used. Fluorescence was visualized on a Leica microscope and data analyzed using Ipwin software (Media Cybernetics, Silver Spring, Maryland).

\section{Statistical Analysis}

The mean and standard error of the mean (SEM) were calculated for the populations studied. To test the null hypothesis that there were no differences between samples of normal bone marrow, CD34+ cells and CD34+ cells from CML patients $\left(\mathrm{H}_{0}\right)$, a two tailed Students $t$-test was applied, and $p$-values $<0.05$ were defined as determining statistical significance.

\section{Results}

\section{FISH Analysis}

CML patients presented in this study were all identified as Bcr-Abl fusion (Philadelphia chromosome)-positive by standard cytogenetics, where $100 \%$ of all metaphase cells scored were found to carry the translocation. To rigorously confirm that the sorted stem and progenitor cells used in this study were Bcr-Abl fusionpositive, fluorescence in situ hybridization (FISH) was performed. We used a highly sensitive method with probes that spanned the common breakpoints of $\mathrm{t}(9 ; 22)(\mathrm{q} 34 ; \mathrm{q} 11.2)$ and that detected double Bcr-Abl fusion in cells with this translocation, one on the abnormal chromosome 9 and one on the Philadelphia chromosome. With this method, the number of false positives and false negatives approaches zero $(24,25)$. FISH analysis was performed on four of the five patients (including one sample of CML bone marrow) and, in each case, the CD34+Lin- cells were shown to be $>95 \%$ BcrAbl fusion-positive. Furthermore, in two of the samples, sufficient CD34+Lin-Thy+ cells were isolated to be scored using this assay, and $>80 \%$ were found to be Bcr-Abl fusion-positive. Using the same assay, no CD34+Lin- cells from normal donors were found to be Bcr-Abl fusion-positive in any of the samples.

\section{Effect of Bcr-Abl Expression on the Maturational Profile of the Stem Cell Compartment}

Subpopulations of stem cells that retain low levels of rhodamine-123 (Rh-123lo) contain the most primitive populations of stem cells defined so far, including quiescent long-term stably repopulating stem cells (26-29). Human CD34+Lin-Thy+Rh-123lo stem cells have been shown to initiate long-term cultures and to repopulate severe combined immunodeficiency (SCID)-Hu mice $(30,31)$. We performed multiparameter flow cytometric analysis to identify such functionally defined viable subpopulations within the CD34+Lin- stem cell compartment (Fig. 1). Using a five parameterbased definition of the CD34 compartment, we were able, for the first time, to define the relative contributions of different subpopulations within CD34+Lin-, according to maturity in CML (Table 1 and Fig. 2A). We found that the proportion of CD34+Lin-Thy + Rh-123lo cells in CML patients was significantly less than that seen in normal cells $(2.3 \pm 0.4, n=5$, and $5.1 \pm 0.6, n=3$, respectively, $p<0.05$ ) (Fig. $2 \mathrm{~A}$ and Table 1). This clearly suggested that acquisition of Bcr-Abl PTK caused some perturbation of this functional compartment, which represents the most primitive phenotypically defined human stem cell. Conversely, we found a significant increase in the proportion of cells with the more mature phenotype of CD34+Lin-Thy-Rh-123hi (high levels of rhodamine-123) in CML, compared with normal samples $(79.3 \pm 1.8, n=5$, and $70.9 \pm 3.3$, $n=3$, respectively, $p<0.05$ ). No significant changes were observed in the CD34+Lin-Thy+ Rh-123hi or CD34+Lin-Thy- Rh-123lo compartments.

Within the CD34+Lin- stem cell compartment, the ratio of primitive progenitors (Thy+Rh-123lo) to more mature cells was increased in CML cells, compared with NBM (Fig. 2B). A ratio of 34.8: 1 CD34+Lin-Thy-Rh123hi cells: CD34+Lin-Thy+Rh-123lo cells 
Table 1. Subpopulation and cell cycle profiles for chronic myeloid leukaemia (CML) and normal bone marrow (NBM) CD34+Lin- stem cells

\begin{tabular}{|c|c|c|c|c|c|c|c|c|}
\hline \multirow[b]{2}{*}{ Sample } & \multicolumn{4}{|c|}{$\%$ of CD34+Lin- } & \multicolumn{4}{|c|}{$\%$ of cells in cycle } \\
\hline & Thy + Rh lo & Thy + Rh hi & Thy-Rh lo & Thy-Rh hi & Thy + Rh lo & Thy + Rh hi & Thy-Rh lo & Thy-Rh hi \\
\hline NBM 1 & 4.0 & 6.0 & 14.5 & 75.5 & 1.3 & 11.2 & 1.1 & 7.1 \\
\hline NBM 2 & 5.8 & 10.5 & 19.3 & 64.3 & 2.8 & 7.4 & 1.7 & 13.0 \\
\hline NBM 3 & 5.5 & 7.6 & 14.1 & 72.7 & 0.5 & 6.2 & 0.3 & 16.0 \\
\hline Mean \pm SEM & $5.1 \pm 0.6$ & $8.0 \pm 1.3$ & $16.0 \pm 1.7$ & $70.9 \pm 3.3$ & $1.5 \pm 0.7$ & $8.3 \pm 1.5$ & $1.0 \pm 0.4$ & $12.0 \pm 2.6$ \\
\hline CML 1 & 3.9 & 7.2 & 13.4 & 75.5 & 2.9 & 11.1 & 2.1 & 7.1 \\
\hline CML 2 & 2.0 & 3.1 & 16.1 & 78.7 & 3.7 & 26.8 & 5.5 & 16.9 \\
\hline CML 3 & 2.0 & 3.1 & 12.7 & 82.3 & 0.5 & 10.9 & 0.5 & 6.7 \\
\hline $\mathrm{CML} 4^{\mathrm{a}}$ & 1.3 & 2.1 & 12.3 & 84.3 & 3.3 & 17.6 & 2.1 & 15.6. \\
\hline CML 5 & 2.2 & 9.5 & 12.8 & 75.5 & 5.4 & 7.1 & 4.5 & 6.4 \\
\hline Mean \pm SEM & $2.3 \pm 0.4^{*}$ & $5.0 \pm 1.4$ & $13.5 \pm 0.7$ & $79.3 \pm 1.8^{*}$ & $3.2 \pm 0.8$ & $14.7 \pm 3.5$ & $2.9 \pm 0.9$ & $10.5 \pm 2.3$ \\
\hline
\end{tabular}

Cell cycle and Rhodamine 123 (Rh-123) staining was performed and analyzed as described in Fig. 1. Results are shown as the individual data profiles and the mean \pm standard error of the mean (SEM) for each population. A Students $t$-test was applied to all the data and significant differences between normal and CML samples are marked $\left({ }^{*} p<0.05\right)$. ${ }^{\mathrm{a}} \mathrm{CML} 4$ is data from a sample of bone marrow.

was seen in CML patients, compared with 13.9: 1 in normal cells, indicating that the CML primitive progenitors underwent approximately one more cell division between the early and the late stages of the stem cell compartment. There was, therefore, overall amplification of the CD34+Lin- compartment, an observation that indicated a change in the self renewal/differentiation rate of CML cells, compared with normal cells. To analyze if this was associated with increased proliferation, we determined the cell cycle status of stem cell compartment subpopulations.

\section{Cell Cycle Status of Bcr-Abl PTK Fusion-Positive CD34+ Lin-stem Cells}

We analyzed highly enriched stem cell populations from Bcr-Abl fusion-positive CML patients to determine if cell cycling status was different in discrete populations of primitive CML stem cells, compared with the same compartments in samples from normal individuals. We found that CD34+Lin- in NBM had only a small proportion (approximately 6-12\%) of cells in cycle when assessed directly on unstimulated cell populations (Fig. 3), reflecting the generally quiescent nature of the stem cell compartment in vivo (32-34). In the CD34+Lincompartment from CML patients, there was a similar proportion in cycle (Fig. 3), compared with normal bone marrow.

Then, we used multicolor flow cytometry to measure five parameters, effectively including cell cycle status and Rh-123 retention (Fig. 1) in CD34+Lin-Thy+ cells. Low Rh-123 staining is thought to reflect reduced mitochondrial activity characteristic of very primitive, quiescent, long-term repopulating stem cells; whereas, higher staining is associated with increased mitochondrial activity in more mature and activated cells $(35,36)$. Our simultaneous analysis of $\mathrm{Rh}-123$ retention and cell cycle on CD34+Lin-Thy+ and CD34+LinThy- populations enabled us, for the first time, to analyze directly the relationship between these functional parameters. We found, as expected, that NBM samples of CD34+LinThy + cells that stained as Rh-123lo were essentially quiescent (Fig. 1, Table 1). Almost no cells were in-cycle $(1.5 \% \pm 0.7, n=3$, incycle); whereas, cells with higher retention of Rh-123 (Rh-123hi) were cycling to a much greater degree $(8.3 \% \pm 1.5, n=3$, in-cycle; Fig. 1 and Table 1). This profile was in keeping with observations from others using sequential staining, where stem cells sorted for low retention of Rh-123 were found to be out of cycle; whereas, cells sorted for high Rh-123 retention had a significant proportion of cells in 

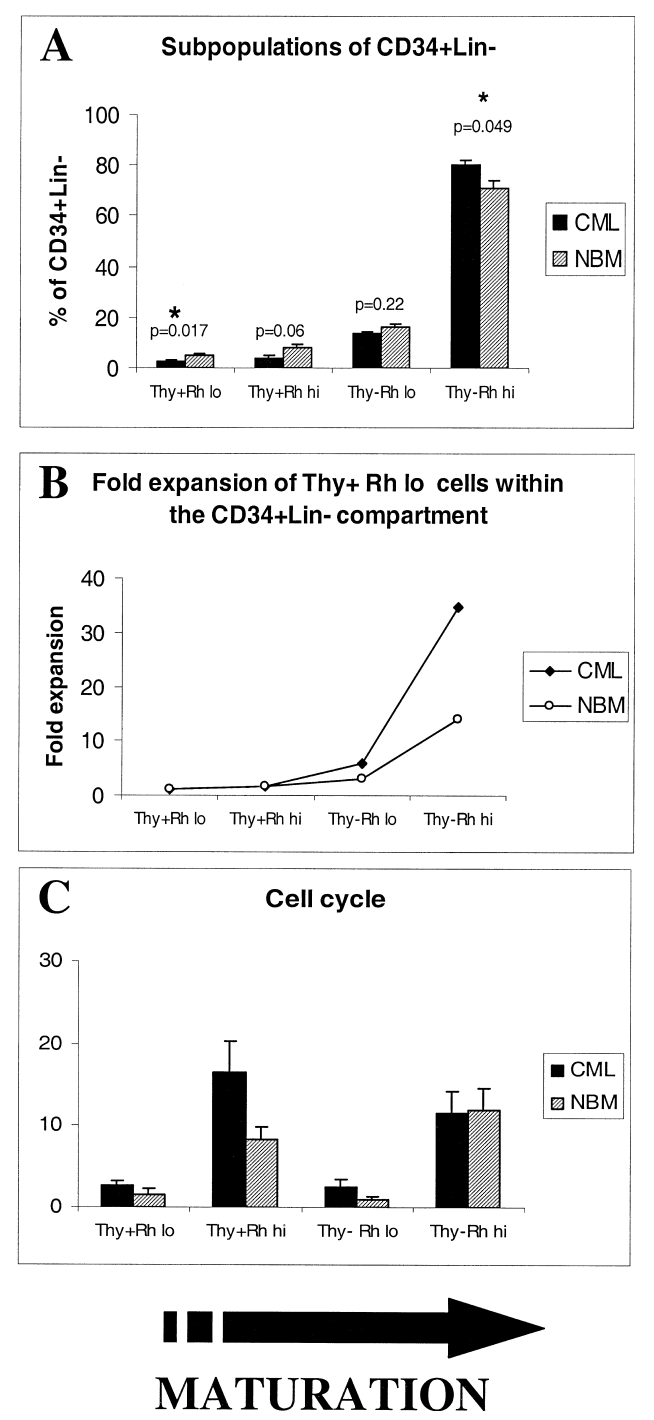

Fig. 2. Effect of Bcr-Abl expression on the maturational profile of the stem cell compartment. The compartments within the CD34+Linstem cell population are shown in order of increasing maturity. (A) The proportion of cells within each compartment for normal bone marrow (NBM; $n=3$ ) and chronic myeloid leukaemia (CML; $n=$ 5), including one bone marrow sample. Results are shown as the mean \pm standard error of the mean (SEM) of the data depicted in Table 1. A Students $t$-test was applied to all the data and $p$-values are shown. Significant differences are marked (*). (B) The fold expansion during maturation was calculated by the ratio of cells in the more mature compartments (CD34+Lin-Thy+Rh-123hi, CD34+Lin-Thy- Rh-123lo and CD34+Lin-Thy-Rh123hi) to those in the initial compartment (CD34+Lin-Thy+Rh-123lo). (C) The proportion of cells in cycle for each population, as determined by Hoechst 33342 staining. Results are shown as the mean \pm standard error of the mean (SEM) for the data depicted in Table 1 . Rh-123lo and $\mathrm{Rh}$ lo, low levels of rhodamine-123; Rh-123hi and Rh hi, high levels of rhodamine-123.

\section{Cycling of committed progenitors}

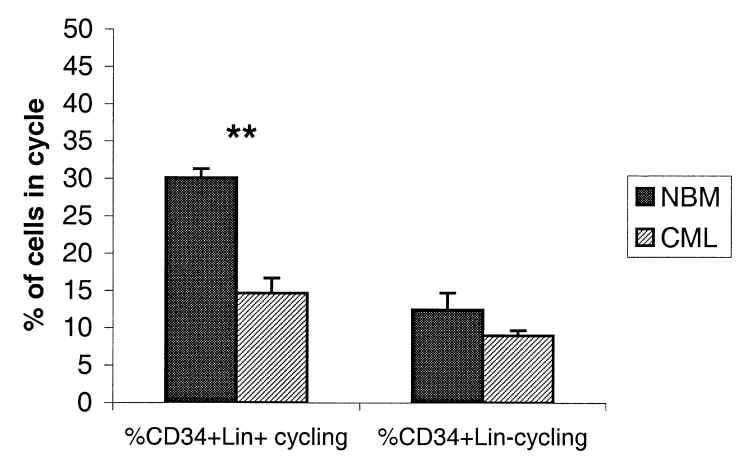

Fig. 3. The cell cycle status of committed progenitors. The percentage of $\mathrm{CD} 34+\mathrm{Lin}+\mathrm{com}-$ mitted progenitor cells in cycle as measured by Hoechst 33342 staining in normal bone marrow $(\mathrm{NBM} ; n=3)$ and chronic myeloid leukaemia (CML; $n=4)$, including two bone marrow samples, is shown. A Students $t$-test was applied to all the data and significant differences are marked $(* * p<0.01)$

cycle $(29,32,37)$. What about the CD $34+$ LinThy + cells from CML samples? These showed a similar overall profile, where cells with low retention of $\mathrm{Rh}-123$ remained largely out of $\mathrm{cy}-$ cle and quiescent (Tablel). The presence of this quiescent, noncycling population within the Bcr-Abl fusion-positive, primitive CML stem cells indicated that acquisition of Bcr-Abl PTK did not necessarily potentiate cell cycling or activation in primitive cells. However, the small proportion of CD34+Lin-Thy + Rh123lo cells in-cycle was higher in cells from CML patients $(3.2 \% \pm 0.8, n=5$, compared with $1.5 \%$ $\pm 0.7, n=3$, in NBM; Table 1, Fig. 2C). This difference in cell cycle status was even more marked in the CD34+Lin-Thy +Rh-123hi population, where $14.7 \% \pm 3.5(n=5)$ of cells were in-cycle, compared with $8.3 \pm 1.5$ in NBM ( $n=3$; Table 1, Fig. 2C). We then extended our observations on Rh-123 staining to the more mature CD34+Lin-Thy- populations. In NBM, $1.0 \% \pm 0.4(n=3)$ CD $34+$ LinThy- Rh-123lo cells were found to be in-cycle, compared with $2.9 \% \pm 0.9(n=5)$ of cells in CML. As was seen in the CD34+Lin-Thy+ compartment, high $\mathrm{Rh}-123$ retention was associated with an increase in the proportion of cells in-cycle in CD34+Lin-Thy- cells, although, in this compartment, similar proportions of cells were found to be in-cycle in NBM and CML $(12.0 \% \pm 2.6$ and $10.5 \% \pm 2.3$, respectively). Although we observed an overall trend of more 
cells cycling in the early compartments of CD34+Lin- populations in CML, compared with NBM, a statistical analysis of the data did not reveal any significant differences. It is possible that an analysis of a large number of samples might bring this to a statistically significant level. However, relatively subtle, but consistent, changes in cell cycling in such early stem cells in CML will have a profound impact on the production of mature cells. We, therefore, proposed that the expansion in the proportion of CD34+Lin-Thy- Rh-123hi in the CD34+Lincompartment (Figs. 2A, 2B) was due largely to marginally increased cycling in the earlier compartments, most markedly within the CD34+ Lin-Thy + Rh-123hi compartment.

\section{Do Committed Progenitor Cells Cycle More Rapidly in CML Patients?}

It might be argued that CML patients displayed the granulocytosis that defines this disease via excessive proliferation in the myeloid-committed progenitor cell compartment. However, the classical studies of Strife, Clarkson and colleagues $(38,39)$ on $\mathrm{CML}$ patients using ${ }^{3} \mathrm{HdT}$ labelling in vivo demonstrated elegantly that granulocyte precursors were cycling more slowly than normal progenitors. To see if we could use our approach to address this issue, we analyzed the cell cycle status of CML and NBM CD34+Lin+, which contained the myeloid-committed progenitor cells. Confirming earlier observations $(38,39)$, we found (Fig. 3) that the CML CD34+Lin+ cells were cycling significantly less than NBM $(14.7 \% \pm$ 2.0, $n=5$, in-cycle in CML, compared with $30.1 \% \pm 2.3, n=3$, in NBM, $p<0.01)$. This analysis included two samples of CML bone marrow cells, to exclude the possibility that the difference was due to the use of peripheral blood rather than bone marrow. Thus, at no stage within the CD34 compartment were there anything more than the most subtle differences in cell cycle between CML and normal samples, until we met the CD34+Lin+ committed progenitors. In this case, CML cells cycle less than normal cells indicating that CML is not a hyperproliferative disorder.

\section{STAT 5 Localization in CML Cells}

Bcr-Abl PTK has been reported to stimulate cells into cytokine-independent growth through activation of the STAT 5 pathway (40), and STAT 5 phosphorylation has been reported to occur in CD34+ Bcr-Abl fusion-positive cells (21). However, as $\mathrm{p} 27^{\mathrm{KIP}}$ is known to be increased in Bcr-Abl fusion-positive cells, yet located in the cytoplasm (where it must be inactive) (41), we decided to determine if STAT 5, a transcription factor, resided within the nucleus of CML progenitors. We, therefore, considered STAT 5 location in the nucleus as a measure of the activity of this protein (42). Peripheral blood mononuclear cells prepared from normal samples and CML samples comprised mainly of mature CD34- cells, many with distinct nuclear morphology typical of myeloid cells (see Fig. 4A-D). In these cells,
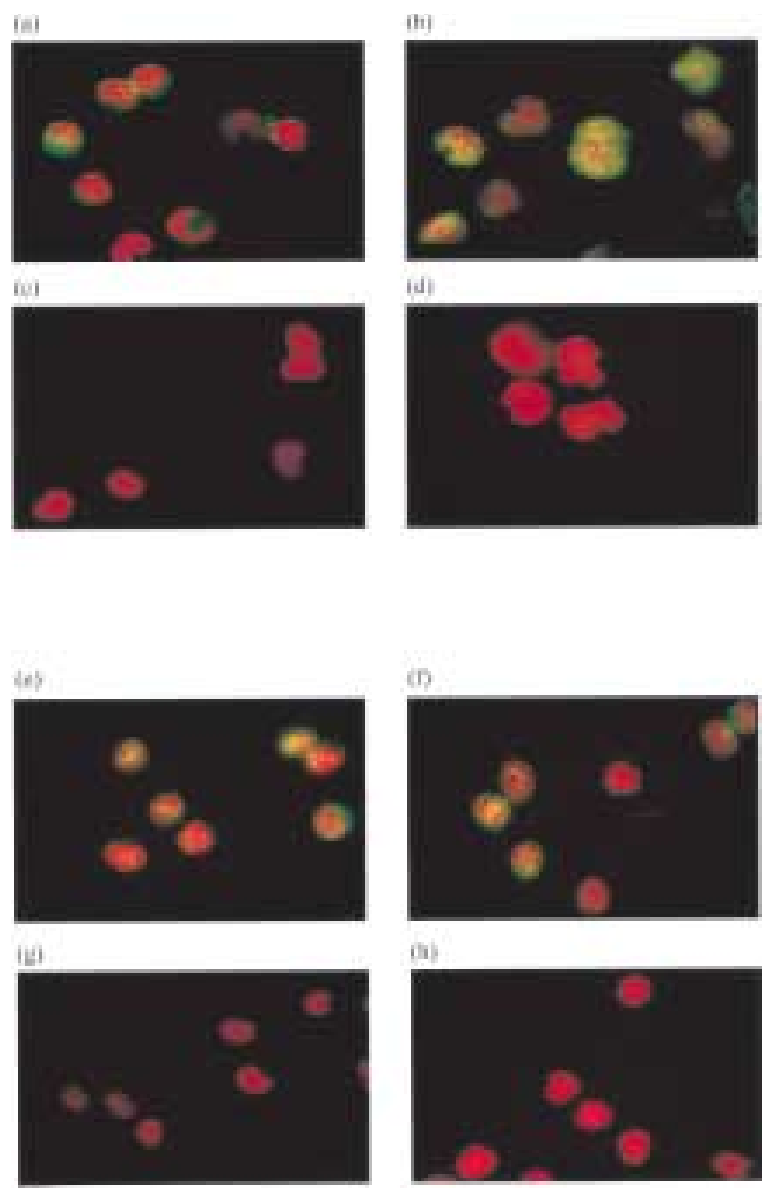

Fig. 4. STAT 5 cellular localization in CD34+ cells and in mononuclear cells from normal bone marrow and chronic myeloid leukaemia (CML) peripheral blood. Propidium iodide staining is depicted in red and (STAT 5) staining in green. Representative STAT 5 antibody staining patterns are shown for normal bone marrow (A) and CML (B) mononuclear cells, and for normal bone marrow CD34+ cells (E) and CML CD34+ cells (F). The respective controls using an irrelevant control antibody are shown in (C) and (D) for mononuclear cells and in $(\mathrm{G})$ and $(\mathrm{H})$ for CD34+ cells. 
there was an increased nuclear localization of STAT 5 in samples from CML patients, compared with samples from normal individuals, where STAT 5 staining was largely cytoplasmic $(n=3$; Fig. $4 \mathrm{~A}-\mathrm{D})$. We also examined STAT 5 translocation in $\mathrm{CD} 4^{+}$cells from healthy individuals $(n=4)$ and CML patients $(n=3)$. All three CML samples were confirmed to be $>95 \%$ Bcr-Abl fusion-positive by FISH. In each sample, there was evidence of some STAT 5 in the nucleus, but no clear difference in STAT 5 nuclear/cytosol ratios between normal and CML samples (Fig. 4E-H).

\section{Discussion}

CML is a complex disease that results from the formation of a Bcr-Abl PTK fusion protein in multipotential bone marrow stem cells. In this study, we rigorously investigated the consequences of acquisition of Bcr-Abl PTK on the maturation and proliferation of the CML haematopoietic stem cell compartment in patients presenting with CML.

We used five-color multiparameter flow cytometry to analyze the most primitive human stem cells identified to date (CD34+LinThy + Rh-123lo), which have been functionally defined by their ability to initiate long-term cultures in vitro and to give long-term reconstitution of SCID mice in vivo $(30,31)$. Our study reveals that there is a significant decrease in the relative proportion of these cells within the CD34+Lin- stem cell compartment, compared with normal cells. This is accompanied by a significant increase in the proportion of mature cells in the later CD34+LinThy-Rh-123hi compartment (Fig. 2A). Thus, we identified distinct and significant perturbations in the maturational hierarchy of the CML stem cell compartment as a consequence of the acquistion of Bcr-Abl PTK. Furthermore, an analysis of the ratio of CD34+Lin-Thy + Rh123lo primitive stem cells to those that enter more mature compartments demonstrates that, in CML, there is an increased expansion during development of the CD34+lin-Thy+Rh123lo subpopulation, compared with normal bone marrow. This must reflect an increased number of cell divisions in CML samples, compared with normal bone marrow during this transition.

We previously showed that activated Abl PTK can interfere with $\left[{ }^{3} \mathrm{H}\right]$-thymidine suicide assays or cytotoxic-drug-induced (such as cytosine arabinoside) cell death (43). This is probably linked to Bcr-Abl-mediated suppression of apoptosis. Unfortunately, both of the above agents are invaluable for cell cycle analysis, but may not be appropriate for the study of CML. In this study therefore, we used a multiparameter flow cytometric assay to directly assess the cell cycle status of cells with Bcr-Abl PTK isolated from patients, and to simultaneously identify subpopulations of cells, such as those with low retention of $\mathrm{Rh}-123$. In this way, we can build a picture of the true extent to which CML is a proliferative disorder and pinpoint the population in the stem cell hierarchy where this proliferation occurs. We found that Bcr-Abl fusionpositive stem cells from patients with CML do not show overt changes in proliferative status, compared with NBM stem cells across the board. The most marked changes were seen in the CD34+Lin-Thy+Rh-123hi compartment (where the cells are undergoing more active proliferation, compared with the heavily quiescent Rh-123lo compartment). In these early two compartments, subtle changes in proliferation and maturation has a potent impact on the size and composition of more mature populations derived from these cells. The small changes in the Rh-123lo and more marked changes in the Rh-123hi populations may, therefore, be the "driving force" for our observed increase in the proportion of mature cells.

A recent study also shows a small (but in this case, statistically significant) decrease in the percentage of cells in G0/G1 in CD34+ stem cells from patients with CML, compared with NBM $(78.1 \%$, compared with $83.5 \%$, respectively) in patients undergoing treatment with hydroxyurea (44). Importantly, the BcrAbl fusion-positive cells used in our study are from patients at presentation, before treatment, and reflect the initial state of the disease without perturbation by treatment with hydroxyurea that may affect the cell cycle status of the stem cells (45). As such, our study gives a better indication of the natural history of CML.

It has been found that CML Bcr-Abl fusion-positive stem cells differ from normal cells in their growth characteristics in longterm cultures where they exhibit decreased self renewal $(46,47)$ and growth factor independence (21). It also has been suggested that most of the leukemic long term culture-initiating cell (LTC-IC) population is in-cycle (48-50).

We now show that the potential for the 
dysregulated growth and proliferation of primitive Bcr-Abl fusion-positive stem cells in culture is not reflected in an overt change in the cell cycle status of the cells as they exist in vivo. Rather, these cells show consistent, subtle changes in the proportion of cells in-cycle, which may have important consequences for expansion in the more mature compartments. We found, however, that changes in the maturational hierarchy do occur within the CD34+Lin- compartment of CML patients in vivo, supporting a role for a change in self renewal/differentiation balance during proliferation. Our experiments also show that acquisition of Bcr-Abl PTK does not perturb the Rh123 staining profiles of haematopoietic stem cells and we suggest that stem cells may remain quiescent in vivo, despite acquisition of Bcr-Abl PTK. Our observations are in keeping with many of the early features of CML, where changes in the stem cell compartment are not clinically manifested for a considerable time and the cells remain, in many respects, phenotypically similar to their normal counterparts. The existence of such a population may also explain the refractoriness of these cells to cellcycle-specific drugs in vivo. It recently has been shown that primitive quiescent $\mathrm{Bcr}-\mathrm{Abl}$ fusion-positive stem cells capable of repopulating SCID mice can be defined (50), supporting the notion that acquisition of Bcr-Abl PTK does not necessarily induce stem cells to activate or proliferate. Our study highlights the importance of examining the consequences of BcrAbl PTK acquisition in the context of the correct cell type, since effects seen in other cells may not necessarily be extrapolated to primary stem cells. Indeed, in the present study, we confirm the original observations of Strife, Clarkson, and colleagues $(38,39)$ that as the Bcr-Abl fusion-positive stem cells mature into committed progenitors they cycle less than their normal counterparts, thus, granulocyte precursors are cycling more slowly than normal progenitors. CML in vivo does appear to be a disease of discordant development.

Phosphorylation of STAT 5 has been associated with Bcr-Abl PTK activation in growth factor-independent CML-derived cell lines and murine bone marrow transformed by $\mathrm{Bcr}-\mathrm{Abl}$ (40), and most recently, in Bcr-Abl fusionpositive primary CD34+ stem cells from CML patients (21). In the current study, we looked at the functional consequence of STAT 5 phosphorylation, translocation to the nucleus, as an indicator of cell activation by Bcr-Abl. We found that mature cells in the peripheral blood of CML patients had evidence of STAT 5 translocation; whereas, normal cells had mainly cytoplasmic staining. STAT 5 phosphorylation has been shown in CD34+ CML stem cells (21) and our data from the CD34+ stem cell compartment suggests that STAT 5 translocation to the nucleus may occur as a consequence of this phosphorylation. Interestingly, translocation was also seen in a proportion of CD34+ progenitors from normal bone marrow, indicating that, as might be expected, STAT 5 translocation is a consequence of normal cytokine-mediated developmental processes in the CD34 compartment.

We, therefore, suggest that the consequence of $\mathrm{Bcr}-\mathrm{Abl}$ expression in vivo is the altering of the self-renewal/maturation balance in primitive stem cells that results in discordant development, as proposed in the classical studies of Strife, Clarkson, and collegues $(38,39)$ rather than overt potentiation of leukaemic cell cycling. Changes in proliferative status as a consequence of Bcr-Abl PTK acquisition are subtle and appear early on, but combined with the change in maturational balance, have dramatic effects on the more mature cells giving rise to the clinical manifestation of CML.

\section{Acknowledgments}

This work was performed at the Leukaemia Research Fund Cellular Development Unit, Department of Biomolecular Sciences, UMIST, Sackville Street, Manchester, M60 1QD, UK. This work was supported by the Leukaemia Research Fund. We would like to thank Jane Owen-Lynch and Clare Murphy for helpful discussions and the members of the Stem Cell Lab at Nottingham City Hospital for their help in obtaining NBM.

\section{References}

1. Konopka J, Watanabe S, Witte O. (1984) An alteration of the human C-Abl protein in $\mathrm{K} 562$ leukemia-cells unmasks associated tyrosine kinase-activity. Cell 37: 1035-1042.

2. Lugo T, Pendergast A, Muller A, Witte O. (1990) tyrosine kinase-activity and transformation potency of Bcr-Abl oncogene products. Science 247: 1079-1082.

3. Daley G, Vanetten R, Baltimore D. (1990) induc- 
tion of chronic myelogenous leukemia in mice by the P210 Bcr/Abl gene of the Philadelphiachromosome. Science 247: 824-830.

4. Elefanty A, Hariharan I, Cory S. (1990) Bcr-Abl, the hallmark of chronic myeloid-leukemia in man, induces multiple hematopoietic neoplasms in mice. $Е M B O ~ J .9:$ 1069-1078.

5. Kelliher M, McLoughlin J, Witte O, Rosenberg N. (1990) induction of a chronic myelogenous leukemia-like syndrome in mice with $\mathrm{V}$-Abl and Bcr/Abl. Proc. Nat. Acad. Sci. U.S.A. 87: 66496653.

6. Gishizky M, Johnsonwhite J, Witte O. (1993) efficient transplantation of Bcr-Abl-induced chronic myelogenous leukemia-like syndrome in mice. Proc. Nat. Acad. Sci. USA 90: 3755-3759.

7. Zhang XW, Ren RB. (1998) Bcr-Abl efficiently induces a myeloproliferative disease and production of excess interleukin-3 and granulocytemacrophage colony-stimulating factor in mice: a novel model for chronic myelogenous leukemia. Blood 92: 3829-3840.

8. Lewis I, McDiarmid L Samels L, To L, Hughes T. (1998) establishment of a reproducible model of chronic-phase chronic myeloid leukemia in NOD/SCID mice using blood-derived mononuclear or CD34(+) cells. Blood 91: 630-640.

9. Carlesso N, Griffin J, Druker B. (1994) use of a temperature-sensitive mutant to define the biological effects of the P210(Bcr-Abl) tyrosine kinase on proliferation of a factor-dependent murine myeloid cell-line Oncogene 9: 149-156.

10. Kabarowski J, Allen P, Wiedemann L. (1994) A temperature-sensitive P210 Bcr-Abl mutant defines the primary consequences of Bcr-Abl tyrosine kinase expression in growth-factor dependent cells $E M B O ~ J . ~ 13: 5887-5895$.

11. Daley G, Baltimore D. (1988) Transformation of an interleukin-3-dependent hematopoietic-cell line by the chronic myelogenous leukemiaspecific p210 Bcr/Abl protein. Proc. Nat. Acad. Sci. U.S.A. 85: 9312-9316.

12. Hariharan IK, Adams JM, Cory S. (1988) Bcr-Abl oncogene renders myeloid cell line factor independent: potential autocrine mechanism in chronic myeloid leukemia. Oncogene Res. 3: 387-399.

13. Laneuville $P$, Sun G, Timm M, Vekemans $M$. (1992) Clonal evolution in a myeloid cell-line transformed to interleukin-3 independent growth by retroviral transduction and expression of p2 $10 \mathrm{Bcr} / \mathrm{Abl}$. Blood 80: 1788-1797.

14. Pierce A, Owen-Lynch PJ, Spooncer E, Dexter TM, Whetton AD. (1998) p210 Bcr-Abl expression in a primitive multipotent haematopoietic cell line models the development of chronic myeloid leukaemia. Oncogene 17: 667-672.

15. Daley G, Ben Neriah Y. (1991) Implicating the $\mathrm{Bcr} / \mathrm{Abl}$ gene in the pathogenesis of Philadelphia chromosome-positive human leukemia. Adv. Cancer Res. 57: 151-184.
16. Gishizky M, Witte O. (1992) Initiation of deregulated growth of multipotent progenitor cells by Bcr-Abl in vitro. Science 25: 836-839.

17. McLaughlin J, Chianese E, Witte O. (1987) In vitro transformation of immature hematopoieticcells by the p210 $\mathrm{Bcr} \mathrm{Abl}$ oncogene product of the Philadelphia-chromosome. Proc. Nat. Acad. Sci. U.S.A. 84: 6558-6562.

18. Scherle P, Dorshkind K, Witte O. (1990) Clonal lymphoid progenitor-cell lines expressing the $\mathrm{Bcr} / \mathrm{Abl}$ oncogene retain full differentiative function. Proc. Nat. Acad. Sci. U.S.A. 87: 19081912.

19. Young J, Witte O. (1988) Selective transformation of primitive lymphoid-cells by the Bcr Abl oncogene expressed in long-term lymphoid or myeloid cultures. Mol. Cell. Biol. 8: 4079-4087.

20. Nishii K, Kabarowski J, Gibbons D, et al. (1996) ts Bcr-Abl kinase activation confers increased resistance to genotoxic damage via cell cycle block. Oncogene 13: 2225-2234.

21. Jiang XY, Lopez A, Holyoake T, Eaves A, Eaves C. (1999) Autocrine production and action of IL-3 and granulocyte colony-stimulating factor in chronic myeloid leukemia. Proc. Nat. Acad. Sci. U.S.A. 96: 12804-12809.

22. Mauer-Satta V, Petzer A, Eaves A, Eaves C. (1996) Bcr-Abl expression in different subpopulations of functionally characterized $\mathrm{Ph}(+)$ CD34(+) cells from patients with chronic myeloid leukemia. Blood 88: 1796-1804.

23. Vandenberg D, Wessman M, Murray L, et al. (1996) Leukemic burden in subpopulations of CD34(+) cells isolated from the mobilized peripheral blood of $\alpha$-interferon-resistant or intolerant patients with chronic myeloid leukemia. Blood 87: 4348-4357.

24. Grand FH, Chase A, Iqbal S, et al. (1998) Twocolor BCR-ABL probe that greatly reduces the false positive and false negative rates for fluorescence in situ hybridization in chronic myeloid leukemia. Genes Chromo. Cancer 23: 109-115.

25. Dewald GW, Wyatt WA, Juneau AL, et al. (1998) Highly sensitive fluorescence in situ hybridization method to detect double BCR/ABL fusion and monitor response to therapy in chronic myeloid leukemia. Blood 91: 3357-3365.

26. Bertoncello I, Hodgson G, Bradley T. (1985) Multiparameter analysis of transplantable hematopoietic stem-cells. The separation and enrichment of stem-cells homing to marrow and spleen on the basis of rhodamine-123 fluorescence. Exp. Hematology 13: 999-1006.

27. Spangrude G, Johnson G. (1990) Resting and activated subsets of mouse multipotent hematopoietic stem-cells. Proc. Nat. Acad. Sci. U.S.A. 87: 7433-7437.

28. Li C, Johnson G. (1995) Murine hematopoietic stem and progenitor cells. Enrichment and biologic characterization. Blood 85: 1472-1479. 
29. Leemhuis T, Yoder MC, Grigsby S, Aguero B, Eder P, Srour E. (1996) Isolation of primitive human bone marrow hematopoietic progenitor cells using hoechst 33342 and rhodamine 123. Exp. Hematology 24: 1215-1224.

30. Baum C, Weissman I, Tsukamoto A, Buckle A, Peault B. (1992) Isolation of a candidate human hematopoietic stem-cell population. Proc. Nat. Acad. Sci. U.S.A. 89: 2804-2808.

31. Uchida N, Combs J, Chen S, Zanjani E, Hoffman R, Tsukamoto A. (1996) Primitive human hematopoietic cells displaying differential efflux of the rhodamine 123 dye have distinct biological activities. Blood 88: 1297-1305.

32. Fleming W, Alpern E, Uchida N, Ikuta K, Spangrude G, Weissman I. (1993) Functional-heterogeneity is associated with the cell-cycle status of murine hematopoietic stem-cells. J. Cell Biol. 122: 897-902.

33. Uchida N, He D, Friera A, et al. (1997) The unexpected G0/Gl cell cycle status of mobilized hematopoietic stem cells from peripheral blood. Blood 89: 465-472.

34. Gothot A, Pyatt R, Mcmahel J, Rice S, Srour E. (1997) Functional heterogeneity of human CD34(+) cells isolated in Subcompartments of the $\mathrm{G}(0) / \mathrm{G}(1)$ phase of the cell cycle. Blood 90: 4384-4393.

35. Ploemacher R, Brons N. (1988) Cells with marrow and spleen repopulating ability and forming spleen colonies on day-16, day-12, and day8 are sequentially ordered on the basis of increasing rhodamine- 123 retention. J. Cell. Phys. 136: 531-536.

36. Kim M, Cooper D, Hayes S, Spangrude G. (1998) Rhodamine-123 staining in hematopoietic stem cells of young mice indicates mitochondrial activa-tion rather than dye efflux. Blood 91: 4106-4117.

37. Wolf N, Kone A, Priestley G, Bartelmez S. (1993) In vivo and in vitro characterization of longterm repopulating primitive hematopoietic-cells isolated by sequential Hoechst-33342- rhodamine123 FACS selection. Exp. Hematology 21: 614-622.

38. Strife A, Clarkson B. (1988) Biology of chronic myelogenous leukemia-is discordant maturation the primary defect? Sem. Hematology 25: 1-19.

39. Clarkson B, Strife A, Perez A, Lambek C, Wisniewski D. (1993) Integration of molecular and biological abnormalities in quest for selective treatment of chronic myelogenous leukemia (CML). Leukemia $\theta$ Lymphoma 11: 81-100.

40. Shuai K, Halpern J, Tenhoeve J, Rao X, Sawyers C. (1996) Constitutive activation of STAT 5 by the Bcr-Abl oncogene in chronic myelogenous leukemia. Oncogene 13: 247-254.

41. Jiang YH, Verfaillie CM. (1999) Inactivation of the cyclin-dependent kinase inhibitor P27 is responsible for overriding integrin engagement mediated inhibition of CML CD34(+) cells. Blood 94: 435.

42. Wilks A, Harpur A. (1994) Cytokine signaltransduction and the Jak family of protein-tyrosine kinases. Bioessays 16: 313-320.

43. Wark G, Heyworth C, Spooncer E, et al. (1998) Abl protein kinase abrogates the response of multipotent haemopoietic cells to the growth inhibitor macrophage inflammatory protein-1 $\alpha$. Oncogene 16: 1319-1324.

44. Traycoff C, Halstead B, Rice S, Mcmahel J, Srour E, Cornetta K. (1998) Chronic myelogenous leukaemia CD34(+) cells exit $G(0) G(1)$ phases of cell cycle more rapidly than normal marrow CD34(+) cells. Brit. J. Haemat. 102: 759-767.

45. Uchida N, Friera A, He D, Reitsma M, Tsukamoto A, Weissman I. (1997) Hydroxyurea can be used to increase mouse C-Kit $(+)$ Thy1.1(Lo)Lin(- /Lo)Sca-1(+) hematopoietic cell number and frequency in cell cycle in vivo. Blood 90: 4354-4357.

46. Eaves C, Cashman J, Eaves A. (1998) Defective regulation of leukemic hematopoiesis in chronic myeloid leukemia. Leuk. Res. 22: 1085-1096.

47. Udomsakdi C, Eaves C, Swolin B, Reid D, Barnett M, Eaves A. (1992) Rapid decline of chronic myeloid leukemic-cells in long-term culture due to a defect at the leukemic stem-cell level. Proc. Nat. Acad. Sci. U.S.A. 89: 6192-6196.

48. Eaves AC, Barnett MJ, Ponchio L, Cashman JD, Petzer A, Eaves CJ. (1998) Differences between normal and CML stem cells: potential targets for clinical exploitation. Stem Cells 16: 77-82.

49. Eaves CJ, Eaves AC. (1987) Cell culture studies in CML. In: Hinton K (ed.) Balliere's Clinical Haematology. Balliere Tindall Saunders, London, pp. 931-945.

50. Holyoake T, Jiang X, Eaves C, Eaves A. (1999) Isolation of a highly quiescent subpopulation of primitive leukemic cells in chronic myeloid leukemia. Blood 94: 2056-206. 